\title{
Determining the Solution Structure of the Drp1 and its Role in the Assembly of the Mitochondrial Fission Machinery
}

\author{
Kristy Rochon ${ }^{1 *}$ and Jason A. Mears ${ }^{1}$
}

1. Department of Pharmacology, Center for Mitochondrial Diseases, the Cleveland Center for Membrane and Structural Biology, Case Western Reserve University School of Medicine, Cleveland, OH, USA.

* Corresponding author:kxr255@case.edu

Mitochondria are double-membrane organelles found in eukaryotic cells and are responsible for cellular metabolism [1]. These organelles undergo fission and fusion in response to cellular stress, and several proteins have been identified to be critical to these processes [2-4]. Dynamin-related protein 1 (Drp1) is the key protein required for mitochondrial fission $[1,5]$. Drp1 is comprised of three conserved domains common to the dynamin family of proteins, the GTPase, middle, and the GTPase effector domain (GED) $[6,7]$. In addition, there is a variable domain (VD), which is intrinsically disordered, that we have shown serves a regulatory function [6-8]. Like dynamin in endocytosis, Drp1 forms dimers [5] that polymerize into helical structures around lipid templates [9]. An atomic structure of Drp1 was resolved using crystallography as part of a larger tetramer in the crystal lattice [10]. However, a mutated construct lacking the VD was used to aid crystallization, and it is unclear whether these changes impact the solution structure of the Drp1. Knowing the native structure of Drp1 before functional polymerization will provide fundamental insight into the early steps of the mitochondrial fission mechanism. This insight could then be used to probe mitochondria dysfunction as it relates to human disease such as neurodegenerative disorders including Huntington's, Parkinson's, and Alzheimer's diseases and amyotrophic lateral sclerosis [11].

We are using electron microscopy studies to resolve structures of the solution form of Drp1 to identify key structural features. Using negative stain microscopy, a sub-20 $\AA$ structure has been resolved of a wild type (WT) and a mutant form of Drp1 (Fig 1a,b). These structures demonstrate a change in conformation of the GTPase domains compared to the crystal structure (Fig 1 c). While this initial finding has identified novel structural features in Drp1, cryoEM imaging is being used to achieve higher resolution. By resolving similar structures of the WT and mutant proteins, we are working to identify samples with maximal homogeneity that preserve native features. Moving forward, we will examine the impact of nucleotide binding on the Drp1 solution structure to study the conformational changes associated with GTP-induced formation of polymers that comprise the minimal constriction machinery.

Overall, our goal is to define the molecular features of Drp1 in solution, which represents the core unit of the mitochondria fission machinery. This pre-assembled state of Drp1 will be compared to assembled polymers to identify conformational changes leading to the formation of the fission machinery. This work also provides a foundation for exploring Drp1 interactions with therapeutic small molecules and peptides [12].

References:

[1] K Labbe, A Murley and J Nunnari, Annu Rev Cell Dev Biol 30 (2014), p. 357.

[2] JY Richard and M v. d. B. Alexander, Science 337 (2012), p. 1062.

[3] JAW. Heymann and JE Hinshaw, J Cell Sci 122 (2009), p. 3427. 
[4] S Gao et al., Nature 465 (2010), p. 502.

[5] PJ Macdonald et al., Mol Biol Cell 25 (2014), p. 1905.

[6] E Smirnova, et al., J Cell Biol 143 (1998), p. 351.

[7] JE Hinshaw, Annu Rev Cell Dev Biol 16 (2000), p. 483.

[8] CA Francy, et al., J Biol Chem 290 (2015), p. 11692.

[9] CA Francy, et al., Scientific reports 7 (2017), p. 3.

[10] C Frohlich et al., The EMBO journal 32 (2013), p. 1280.

[11] E Lezi and RH Swerdlow, Adv Exp Med Biol 942 (2012), p. 269.

[12] The authors acknowledge funding from the National Institute of Health (NIH 1R01GM125844-01), imaging and sample screening support from Harry Scott, Wei Huang, and Derek Taylor.

A 2D Classification
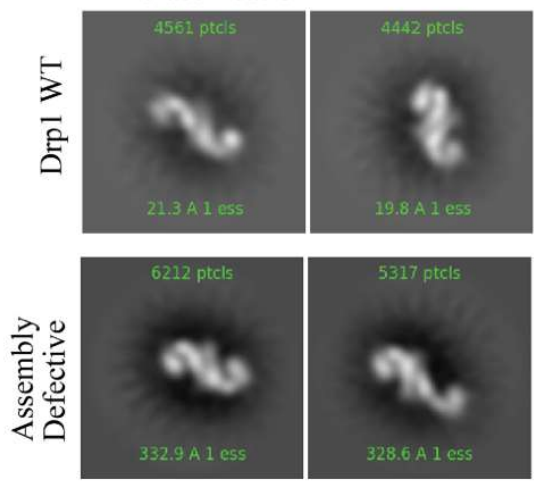

B

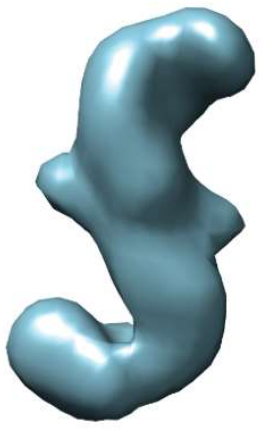

WT
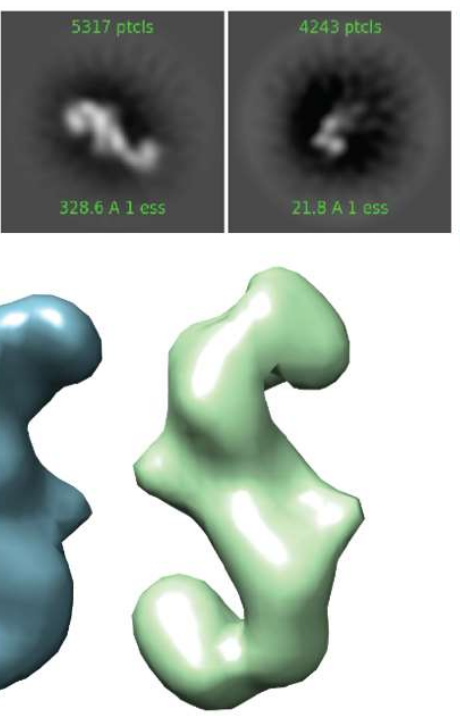

Assembly Defective

14,292 Particles

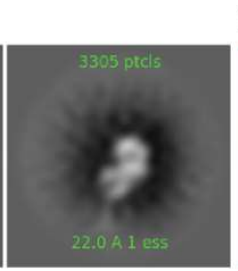

3D Classification

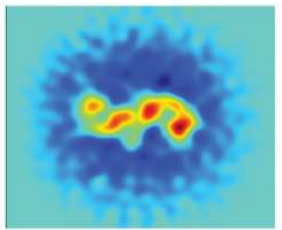

Maximum Resolution
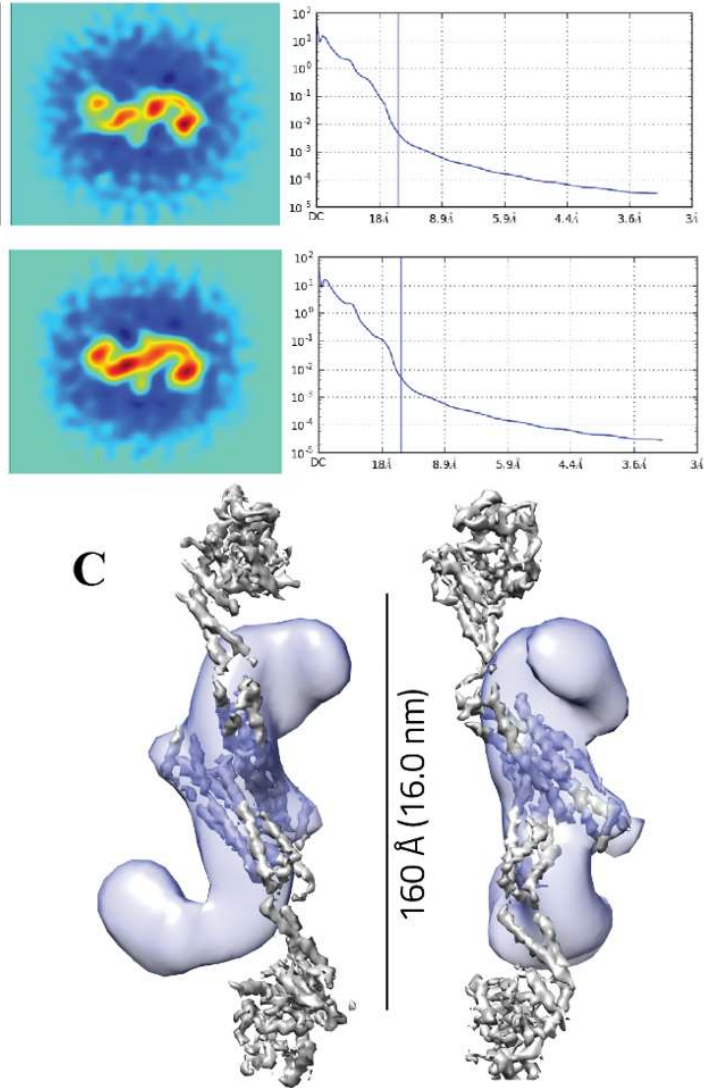

Figure 1. (a) Using negative stain electron microscopy, the solution structure of Dynamin-related protein 1 (Drp1) was resolved to a sub-20 Å resolution. (b) The mutant dimer was used to improve homogeneity, and comparison with wild type dimers highlights similarities that validate the use of the assembly-incompetent mutant in future studies. (c) Docking the crystal structure 4BEJ in the negative stain reconstruction demonstrates a unique conformation due to flexibility in the sequence adjacent to the GTPase domain. 